\title{
Overexpressed cyclin D3 contributes to retaining the growth inhibitor p27 in the cytoplasm of thyroid tumor cells
}

\author{
Gustavo Baldassarre, ${ }^{1}$ Barbara Belletti, ${ }^{1}$ Paola Bruni, ${ }^{1}$ Angelo Boccia, ${ }^{1}$ \\ Francesco Trapasso, ${ }^{2}$ Francesca Pentimalli, ${ }^{1}$ Maria Vittoria Barone, ${ }^{1}$ \\ Gennaro Chiappetta, ${ }^{1}$ Maria Teresa Vento, ${ }^{1}$ Stefania Spiezia, ${ }^{1}$ Alfredo Fusco, ${ }^{2}$ \\ and Giuseppe Viglietto ${ }^{1}$
}

${ }^{1}$ Istituto Nazionale dei Tumori "Fondazione Senatore Pascale," 80131 Naples, Italy

${ }^{2}$ Dipartimento di Medicina Sperimentale e Clinica, Facoltà di Medicina e Chirurgia di Catanzaro,

Università degli Studi di Reggio Calabria, Calabria, Italy with Università degli Studi di Cantanzaro, 88100, Cantanzaro, Italy

Address correspondence to: Giuseppe Viglietto, Oncologia Sperimentale E, Istituto Nazionale dei Tumori,

Via M. Semmola, 80131 Naples, Italy. Phone: 081-590-3549; Fax: 081-590-3838; E-mail: gvigliet@tin.it.

Received for publication February 2, 1999, and accepted in revised form August 17, 1999.

The majority of thyroid carcinomas maintain the expression of the cell growth suppressor p27, an inhibitor of cyclin-dependent kinase-2 (Cdk2). However, we find that $80 \%$ of $\mathrm{p} 27$-expressing tumors show an uncommon cytoplasmic localization of p27 protein, associated with high Cdk2 activity. To reproduce such a situation, a mutant $\mathrm{p} 27$ devoid of its $\mathrm{COOH}$-terminal nuclear-localization signal was generated (p27-NLS). p27-NLS accumulates in the cytoplasm and fails to induce growth arrest in 2 different cell lines, indicating that cytoplasm-residing p27 is inactive as a growth inhibitor, presumably because it does not interact with nuclear Cdk2. Overexpression of cyclin D3 may account in part for p27 cytoplasmic localization. In thyroid tumors and cell lines, cyclin D3 expression was associated with cytoplasmic localization of p27. Moreover, expression of cyclin D3 in thyroid carcinoma cells induced cytoplasmic retention of cotransfected p27 and rescued p27-imposed growth arrest. Endogenous p27 also localized prevalently to the cytoplasm in normal thyrocytes engineered to stably overexpress cyclin D3 (PC-D3 cells). In these cells, cyclin D3 induced the formation of cytoplasmic p27-cyclin D3-Cdk complexes, which titrated p27 away from intranuclear complexes that contain cyclins A-E and Cdk2. Our results demonstrate a novel mechanism that may contribute to overcoming the p27 inhibitory threshold in transformed thyroid cells.

J. Clin. Invest. 104:865-874 (1999).

\section{Introduction}

Thyroid neoplasms originating in follicular cells comprise a broad spectrum of tumors with a wide variety of biological and clinical phenotypes, and therefore represent a good model of multistage epithelial tumorigenesis (1).

Tumor development results from genetic alterations that affect genes involved in the regulation of cell growth and differentiation $(2,3)$. Inactivation of tumor-suppressor genes as well as mutational activation of oncogenes is believed to lead to clonal expansion of genetically modified cells (2). The different biological and clinical phenotypes of thyroid tumors have been associated with specific genetic alterations involving oncogenes (e.g., ras, RET/PTC) $(4,5)$ and tumor suppressor genes (e.g., p16 and p53) (6). However, although tumor development is characterized by loss of restraints on cell growth and by progressive impairment of differentiation, the roles of the individual components of the cell-cycle machinery in thyroid tumorigenesis remain to be determined.

In mammalian cells, factors that determine whether cells proliferate or growth is arrested operate during the G1 phase of the cell cycle $(7,8)$. G1 progression is controlled by interactions among 3 families of pro- teins: cyclin-dependent kinases (Cdk's); their activating partners, the $\mathrm{G} 1$ cyclins; and inhibitory proteins known as Cdk inhibitors $(9,10)$. So far, 2 classes of Cdk inhibitors have been identified: the INK4 proteins that are specific inhibitors of cyclin $\mathrm{D}-\mathrm{Cdk} 4$ and cyclin D-Cdk6, and the Kip/Cip inhibitors which, inhibit all cyclin-Cdk complexes (10). The Kip/Cip family of Cdk inhibitors comprises p21, p27, and p57 (11-13).

Because it induces growth arrest (12) and p27-deficient mice are prone to develop tumors (14), $p 27$ represents a potential tumor-suppressor gene. However, in contrast to traditional antioncogenes such as $p 15$ and $p 16$, rare mutations in the $p 27$ gene have been reported in human tumors. Nevertheless, the finding that p27 expression is reduced in several tumors suggests that p27 may have an important role in human carcinogenesis (15-17). Accordingly, 2 studies reporting reduced p27 expression in thyroid tumors have been published $(18,19)$. However, it has become clear that p27 subcellular localization may have a relevant role in its function (20). Therefore, we performed analysis of $\mathrm{p} 27$ expression accompanied by a careful determination of its localization in a panel of thyroid carcinoma biopsies and tumor-derived cell lines, and addressed the significance of this localization. 


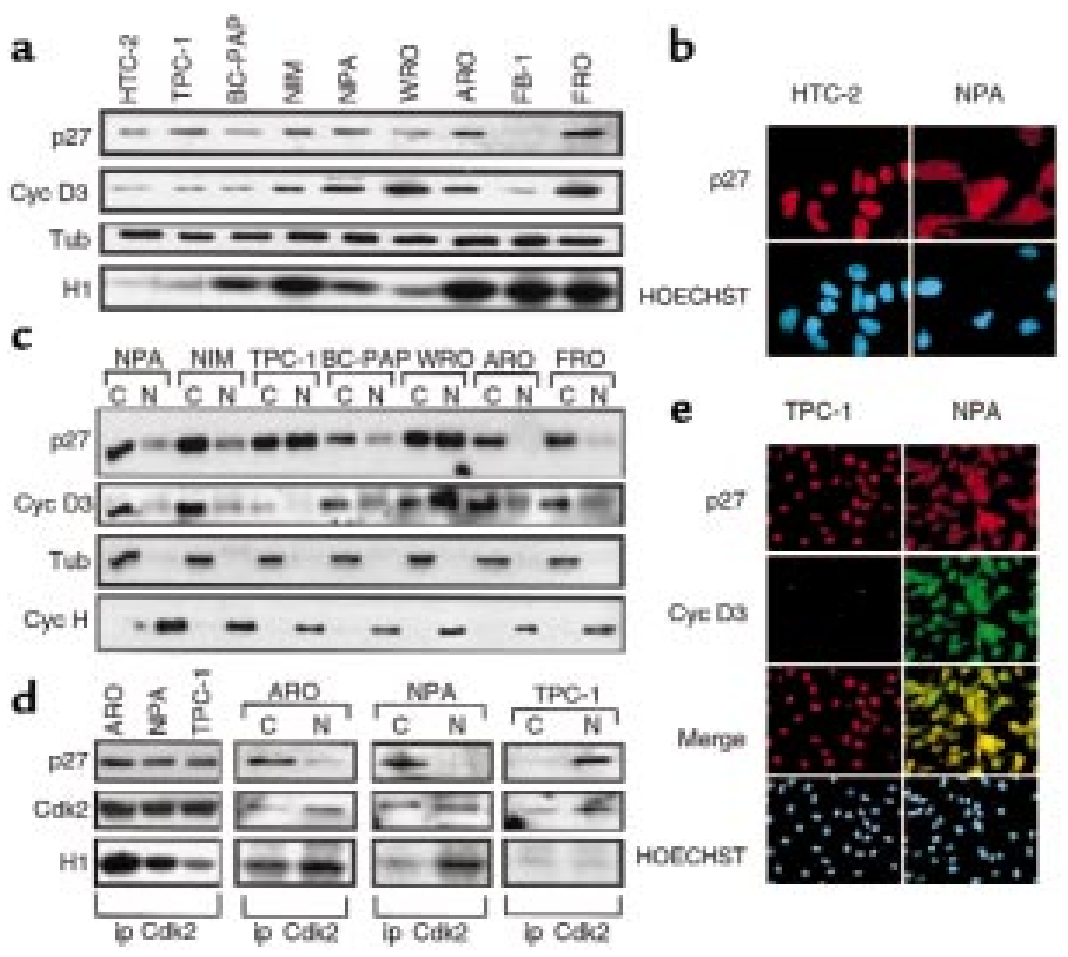

\section{Figure 1}

Expression and localization of p27 and cyclin D3 (Cyc D3) in thyroid carcinoma-derived cell lines. (a) Western blot analysis of p27 and cyclin D3 expression in thyroid tumor-derived cell lines. $\alpha$-tubulin (Tub) was used to assure uniform loading (third row). Bottom row: Cdk2 activity in protein extracts from the same cell lines using histone $\mathrm{H} 1$ as substrate. (b) Immunofluorescence analysis of p27 expression, $\times 100$. (c) Western blot analysis of p27 and cyclin D3 on differentially fractionated proteins (C, cytoplasmic fractions; $\mathrm{N}$, nuclear fractions). As control, antibodies against $\alpha$-tubulin or cyclin $\mathrm{H}$ were used. (d) Cdk2 immunoprecipitates: p27 bound to Cdk2, Cdk2 level, and Cdk2 activity in total (left column) or fractionated extracts (remaining columns). (e) Colocalization of p27 and cyclin D3 in thyroid tumor cell lines.

\section{Methods}

Cell lines. The human cell lines used in this study are described in ref. 21. Bosc23 cells were a gift of M. Santoro (Consiglio Nazionale delle Ricerche, Naples, Italy). All cell lines were grown in DMEM containing 10\% FCS. PC Cl 3 and PC-D 3 cells (normal thyrocytes engineered to stably overexpress cyclin D3) were grown in Ham's F12 medium supplemented with $5 \%$ calf serum in the presence of 6 hormones (thyrotropin, hydrocortisone, insulin, transferrin, somatostatin, and glycyl-histidyllysine; Sigma Chemical Co., St. Louis, Missouri, USA).

Immunoperoxidase staining. Immunohistochemistry was performed using anti-p27 monoclonal antibody k25020 (Transduction Laboratories, Lexington, Kentucky, USA) or anti-p27 polyclonal antibody C-19 (Santa Cruz Biotechnology Inc., Santa Cruz, California, USA) as described previously $(15,16)$. Antigen retrieval was performed by microwave irradiation. To define $\mathrm{p} 27$ expression we used cutoff values that have been defined in previous papers $(15,16)$. Tumors were considered to be p27-positive when $50 \%$ or more of the tumor cells stained positive; if less than $50 \%$ of cells stained positive, a tumor was considered p27-negative. Counts were performed in 5 random high-power fields. At least 500 cells were counted.

Western blotting, immunoprecipitation, and kinase assay. Cells were lysed in NP-40 buffer containing protease inhibitors. Proteins were separated on polyacrylamide gels and transferred to nitrocellulose membranes (Hybond-C; Amersham Pharmacia Biotech, Uppsala, Sweden). Membranes were incubated with primary and secondary antibodies and revealed by enhanced chemiluminescence (Amersham Pharmacia Biotech). Differential extraction of nuclear or cytoplasmic proteins was performed as reported previously (22). Immunoprecipitation and kinase assays were performed as described (23).

Constructs and transfection. The p 27 constructs have been described (23). p27-NLS: forward primer, nucleotides 1-21; reverse primer, nucleotides 453-432 (12). p27-97197: forward primer, nucleotides 287-312; reverse primer, nucleotides 576-597. p27-1-186: forward primer, nucleotides 1-21; reverse primer, nucleotides 538-558. The mutant p27-TA187 was obtained by use of a sitespecific mutagenesis kit (Roche Molecular Biochemicals, Mannheim, Germany). Cyclin D3 was obtained by RTPCR using primers 166-187 and 1023-1044, which were designed according to ref. 24 . The correct DNA sequence was confirmed by DNA sequencing. FB-1 and PC $\mathrm{Cl} 3$ cells were transfected by electroporation. Bosc 23 cells were transfected by the calcium phosphate procedure.

Indirect immunofluorescence analysis. Cells were grown to subconfluence on coverslips, fixed in 3\% paraformaldehyde, and permeabilized with $0.2 \%$ Triton X-100. Secondary antibodies conjugated with Texas red or FITC were used to reveal p27- and cyclin D3-positive cells. Cell nuclei were identified by HOECHST staining. Fluorescence was viewed with a Zeiss 140 epifluorescent microscope equipped with filters that allowed discrimination between Texas red and FITC.

5'-bromo-3'-deoxyuridine incorporation. Transfection of 5 $\times 10^{5}$ cells was performed. Twenty-four hours later, $5^{\prime}-$ bromo-3'-deoxyuridine (BrdU) was added to the culture medium to a final concentration of $10 \mu \mathrm{M}$ and allowed to react for 1 hour. Detection of eukaryotic green flourescent protein (EGFP) and BrdU was performed as described (24). A 5'-bromo-3'-deoxyuridine Labeling 
and Detection Kit from Boehringer Mannheim was used to identify S-phase cells.

Antibodies. The antibodies used were C-19 (anti-p27), M2 (anti-Cdk2), M20 (anti-cyclin E), C-16 (anti-cyclin D3), C-22 (anti-Cdk4), C-21 (anti-Cdk6), and f-7 (anti-influenza hemagglutinin protein [anti-HA]) from Santa Cruz Biotechnology Inc., and k25020 (anti-p27) and anti-Cdk2 from Transduction Laboratories.

\section{Results}

Expression of p27 and cyclin D3 in thyroid tumors and tumor-derived cell lines. We analyzed p27 expression in 8 cell lines derived from human thyroid tumors. Four lines were derived from papillary carcinomas (TPC-1, BC-PAP, NIM, and NPA), 1 from follicular carcinomas (WRO), and 3 from anaplastic carcinomas (ARO, FB-1, and FRO). The primary HTC-2 cells were used as control normal thyrocytes. Western blot analysis demonstrated that p27 expression was lost in the anaplastic cell line FB-1, whereas it was retained in all other lines (Figure 1a). Extracts from thyroid carcinoma-derived cell lines were assayed for activity of Cdk2 (a major inhibition target of p27) using histone $\mathrm{H} 1$ as a substrate (Figure 1a, bottom). Surprisingly, we failed to find any significant correlation between the level of $\mathrm{p} 27$ protein and the activity of Cdk2; in most tumor cell lines, Cdk2 activity was increased independently of p27 expression.

We also analyzed p27 expression by Western blot in normal thyroid tissue (4 biopsies) and in 45 primary thyroid carcinomas (28 papillary, 9 follicular, and 8 anaplastic carcinomas). Results are summarized in Table 1. In normal thyroid, p27 was detected at high levels. Conversely, p27 protein was reduced in 10 of 28 papillary carcinomas, in 3 of 9 follicular carcinomas, and in 6 of 8 anaplastic carcinomas. In the remaining tumors, p27 was expressed at levels similar to those in normal thyroid. A representative Western blot is shown in Figure 2a, showing that p27 is expressed at high levels in normal thyroid gland (lane NT) and in some tumors (lanes 2,4 , and 6-8), but is downregulated in other tumors (lanes 1, 3, 9, and 10). Tumor extracts were assayed for Cdk2 activity (Figure 2a, bottom). $\mathrm{Cdk} 2$ activity was higher in carcinomas than in normal thyroid tissue; increased Cdk2 activity was also detected in tumors with high p27 expression (lanes 2, 6, and 8). Expression of p27 was confirmed in 27 representative carcinomas by immunostaining. Determination of p27 expression was performed as described in Methods. A good correlation was found between Western blot and immunostaining results.

Subcellular localization of p27 in thyroid tumor cell lines and in normal and neoplastic thyroid tissue. Because the subcellular localization of $\mathrm{p} 27$ has been found to be altered in some tumors $(25,26)$, we investigated whether changes in the cellular compartment in which p27 resides could account for the lack of its inhibitory activity observed in thyroid tumors and tumor cell lines. As determined by indirect immunofluorescence, in normal thyrocytes p27 resided almost exclusively in the nucleus (more than $70 \%$ of HTC- 2 cells showed nuclear staining). In BCPAP, WRO, and TPC- 1 cells, p27 resided in the nucleus and in the cytoplasm (30-60\% of cells showed nuclear staining). Conversely, less than $15 \%$ of NIM, NPA, FRO, and ARO cells showed nuclear p27, with most cells showing diffuse cytoplasmic or nucleocytoplasmic staining. (See Figure 1b for TPC-1 and NPA cells; data are not shown for the cell lines.)

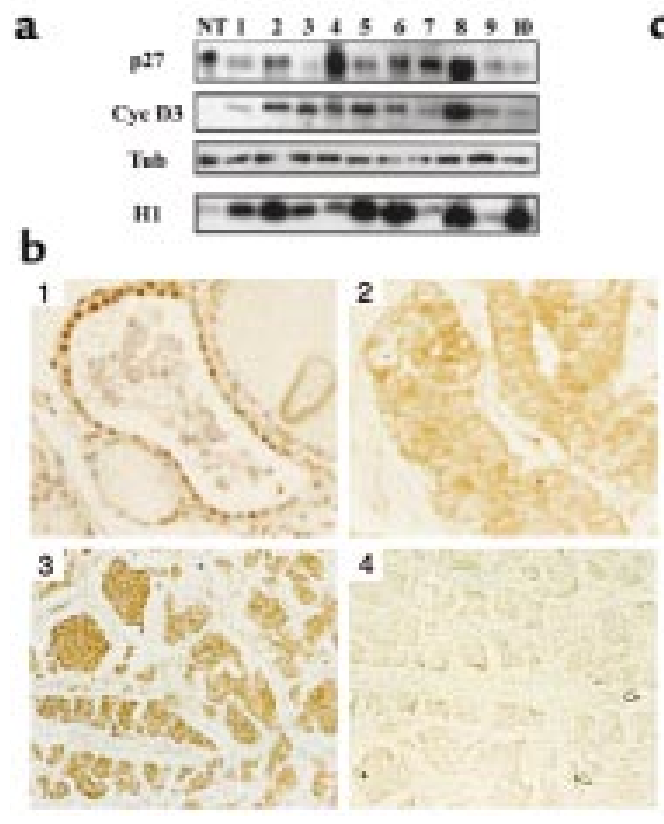

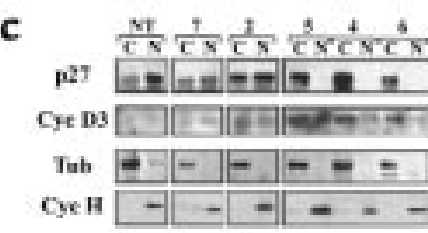

d

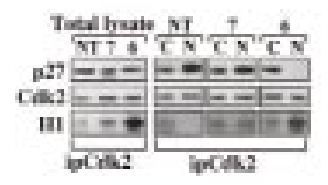

e

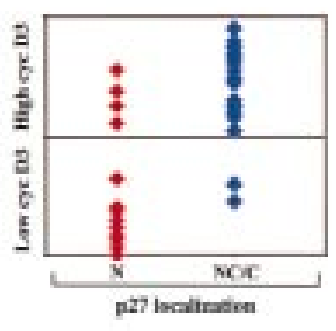

\section{Figure 2}

Expression and localization of p27 and cyclin D3 in thyroid tumors. (a) Western blot analysis of p27 or cyclin D3 expression in thyroid tumors. Lane NT, normal thyroid; lanes 1-5, papillary carcinomas; lanes 6 and 7, follicular carcinomas; lanes 8-10, anaplastic carcinomas. $\alpha$-tubulin was used to assure uniform loading (third row). Bottom, Cdk2 activity in protein extracts from the same samples. (b) p27 immunoreactivity in normal and neoplastic thyroid tissue. Top left: normal thyroid, nuclear $\mathrm{p} 27$. Top right: papillary carcinoma, cytoplasmic p27. Bottom left: papillary carcinoma, nuclear and cytoplasmic p27. Bottom right: same tumor with antibody preincubated with antigen. $\times 40$. (c) Western blot analysis of p27 and cyclin D3 on differentially extracted proteins. As control, antibodies to $\alpha$-tubulin or cyclin $\mathrm{H}$ were used. (d) Cdk2 immunoprecipitates: 27 bound to Cdk2, Cdk2 level, and Cdk2 activity in total (left column) or fractionated extracts (remaining columns). (e) Correlation between cyclin D3 expression and p27 localization. Data are from Table 1. 


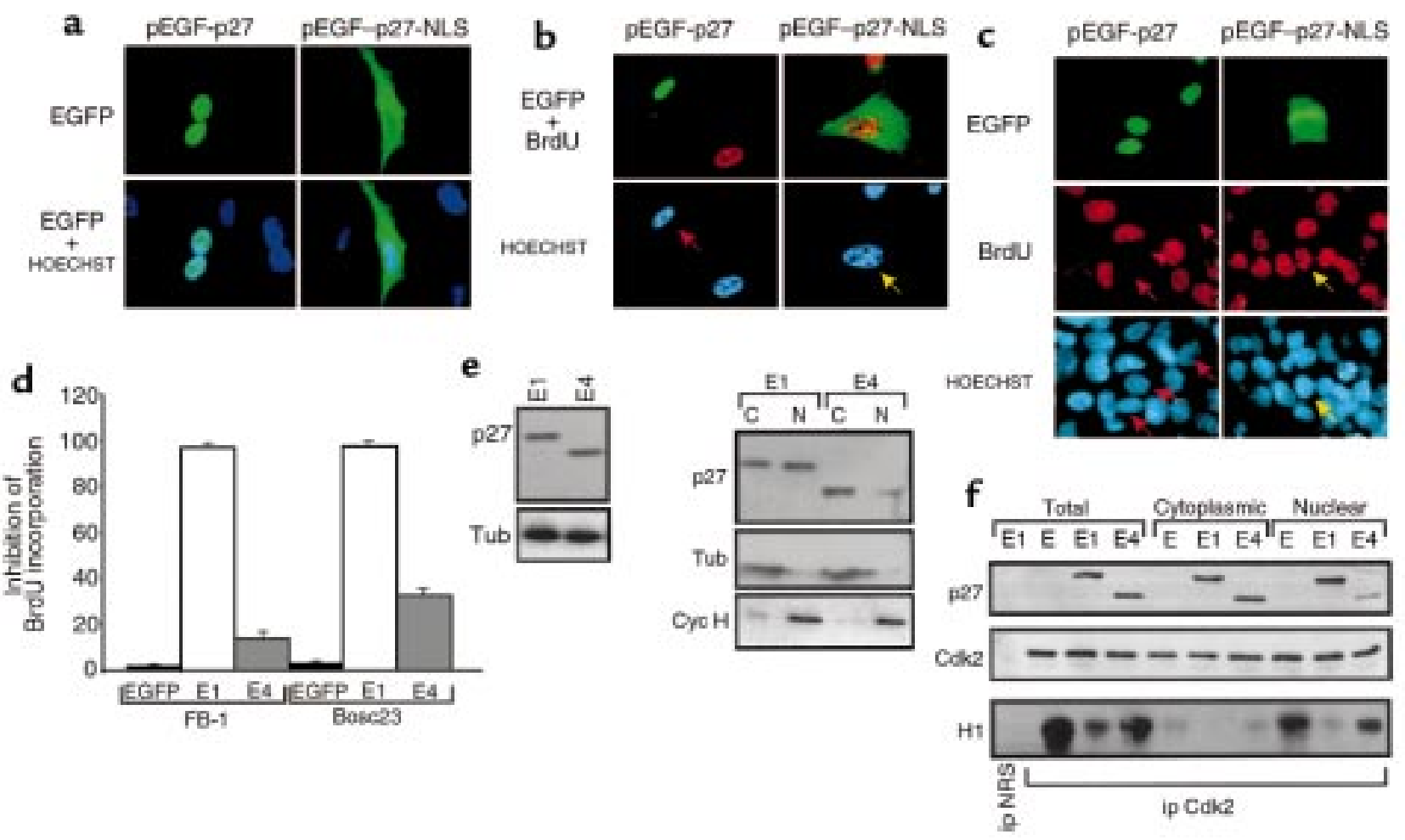

Figure 3

Nuclear localization of p27 is required for p27 activity. (a) Localization of pEGF-p27 and pEGF-p27-NLS in FB-1 cells. Green fluorescence of EGFP was used to identify the localization of fused p27 (top) or was merged with HOECHST staining of nuclei (bottom). (b) BrdU incorporation of FB-1 cells transfected with pEGF-p27 or pEGF-p27-NLS. Left: red arrow shows a pEGF-p27-transfected cell that is not incorporating BrdU. Right: yellow arrow shows a cell transfected with pEGF-p27-NLS incorporating BrdU. (c) BrdU incorporation of Bosc23 cells transfected with p27 or p27-NLS. Green fluorescence of the EGFP protein was used as a flag to identify the localization of fused p27 (top). Middle: BrdU staining. Bottom: HOECHST staining. Left column: red arrows show pEGF-p27-transfected cells that have not incorporated BrdU. Right column: yellow arrow shows a cell transfected with pEGF-p27-NLS and incorporated BrdU. A Neo-Achromat Zeiss objective lens $(\times 100)$ was used. (d) Statistical analysis of inhibition of BrdU uptake in FB-1 and Bosc23 cells transfected with p27 or p27-NLS. Data represent the mean \pm SD of 3 different experiments performed in duplicate; at least 200 cells were counted. (e) Plasmid expression (left) and localization (right) in Bosc23 cells. (f) Cdk2 immunoprecipitates in Bosc23 cells: p27 bound to Cdk2, Cdk2 level, and Cdk2 activity in total or fractionated extracts. E, EGFP; E1, wild-type pEGF-p27; E4, pEGF-p27-NLS.

Subcellular localization of p27 was confirmed by Western blot on extracts enriched with cytoplasmic and nuclear proteins. In HTC-2 cells, p27 was essentially nuclear. It was detected in the nuclear and cytoplasmic compartments in WRO and TPC-1 cells, but was limited to the cytoplasmic compartment in NPA, NIM, FRO, and ARO cells (Figure 1c). The degree of crosscontamination was determined by the use of control antibodies - anti- $\alpha$-tubulin antibodies as control for cytoplasmic proteins and anti-cyclin $\mathrm{H}$ antibodies as control for nuclear proteins.

Localization of $\mathrm{p} 27$ was also analyzed in normal thyroid tissue (4 biopsies) and in 27 representative carcinomas (18 papillary, 5 follicular, and 4 anaplastic carcinomas). For immunoperoxidase staining we used 2 different anti-p27 antibodies (k25020 and C-19), and obtained similar results. Immunoreactivity of p27 was detected in the nuclei of most follicular cells in normal thyroid gland ( $69 \pm 12 \%$ ), with most cells presenting as intensely positive. Immunostaining for p27 in thyroid carcinomas showed high variability. Immunoreactivity was low or absent in 3 of 18 papillary carcinomas, 1 of 5 follicular carcinomas, and 2 of 4 anaplastic carcinomas. A high percentage of $\mathrm{p} 27$-positive cells (more than $50 \%$ ) was found in the remaining carcinomas. Among these, p27 immunoreactivity was predominantly nuclear in 5 biopsies (of 4 papillary and 1 follicular carcinoma), and both nuclear and cytoplasmic in 5 biopsies (of papillary carcinomas). The remaining 11 carcinomas displayed a predominant diffuse cytoplasmic p27 immunoreactivity. In total, in 16 of 21 thyroid tumors with high p27 expression, p27 was found to reside in an uncommon compartment. A representative case is shown in Figure 2b. In nonneoplastic thyrocytes, p27 is nuclear (Figure 2b, lane 1), but in a papillary carcinoma it is restricted to the cytoplasm (Figure 2b, lane 2). In this case, counterstaining with hematoxylin was omitted to appreciate empty nuclei of tumor cells. Cytoplasmic staining was observed with both antibodies and was competed by recombinant hexahistidinetagged $\mathrm{p} 27$. Figure $2 \mathrm{~b}$ (lane 3 ) shows a papillary tumor stained with anti-p27 and a parallel section of the same 
tumor in which the antibody was preincubated with a 10 -fold molar excess of the antigen.

Subcellular localization of p27 was confirmed by Western blot. Cytoplasm- and nuclear-enriched proteins were prepared from 2 normal thyroids, 1 tumor with predominant $\mathrm{p} 27$ nuclear staining, 4 tumors with both nuclear and cytoplasmic p27 staining, and 5 tumors with essentially cytoplasmic p27 staining. Western blot results confirmed data from immunostaining. Almost $70 \%$ of p27 was found in the nuclear compartment in normal thyroid gland and in the tumor with nuclear staining; a variable fraction ranging from $25-60 \%$ of p 27 was found in the nuclear compartment in tumors with mixed localization. In tumors that, by immunostaining, had demonstrated cytoplasmic localization, levels of nuclear p27 were undetectable (Figure $2 \mathrm{~b}$, lanes 5,4 , and 6 , respectively). A representative experiment is presented in Figure $2 c$. Antibodies to $\alpha$-tubulin and cyclin $\mathrm{H}$ were used to assess the quality of the subcellular fractionation.

Cdk2 activity correlates with the level of $p 27$ bound to nuclear Cdk2 in thyroid tumors and tumor-derived cell lines. In most tumors and tumor-derived cell lines, cytoplasmic p27 localization was associated with high Cdk2 activity (compare TPC-1 and WRO cells with NPA, ARO, and FRO cells in Figure 1, a and c; compare lanes 4-6 with lane 7 in Figure 2, a and c). Therefore we investigated whether the high Cdk2 activity observed in tumors or cell lines that retained p27 expression could be due to the fact that p27 in cytoplasm had no access to nuclear $\mathrm{Cdk} 2$. To determine this, $\mathrm{Cdk} 2$ was immunoprecipitated from total or differentially fractionated HTC-2 and tumor-derived cell lines. One-tenth of the immunoprecipitate was used for kinase assay; the remainder was used to determine the levels of Cdk2-bound p27. Finally, blots were reprobed with anti-Cdk2 to normalize the amount of Cdk 2 immunoprecipitated. Figure $1 \mathrm{~d}$ shows the results obtained with 3 representative cell lines (ARO and NPA for cytoplasmic p27; TPC-1 for nuclear $\mathrm{p} 27)$. Approximately the same level of $\mathrm{p} 27$ was bound to $\mathrm{Cdk} 2$ in total extracts in ARO, NPA, and TPC- 1 cells, but $\mathrm{Cdk} 2$ activity was higher in ARO and NPA cells than in TPC-1 cells (Figure 1d, left). When we analyzed differentially fractionated extracts, we found that in ARO and NPA cells, little p27 was bound to nuclear Cdk2, whereas in TPC- 1 cells most $\mathrm{p} 27$ was bound to nuclear $\mathrm{Cdk} 2$ (right). It is of note that in all tumor-derived cell lines, Cdk 2 activity was essentially nuclear. Therefore, it appears that in thyroid tumor-derived cell lines, total Cdk2 activity is inversely correlated with the amount of p27 bound to nuclear Cdk2. Similar results were obtained from the analysis of 10 surgically removed tumors (Figure 2d, compare lanes NT, 7, and 6).

Expression and localization of cyclin D3 in thyroid tumor cell lines. Recent studies have reported the existence of a tight correlation between D cyclins and p27 expression in colon carcinomas (25). Among the D cyclins, cyclin D3 is the most likely to be involved in neoplastic transformation of thyroid cells, because it is required for the

\section{Table 1}

Correlation between cyclin D3 expression and p27 localization in thyroid tumors and cell lines

\begin{tabular}{lccccc}
\hline \multirow{2}{*}{ Histology } & \multicolumn{2}{c}{ Expression $^{\mathrm{A}, \mathrm{B}}$} & \multicolumn{3}{c}{ Localization $^{\mathrm{C}}$} \\
& Cyclin D3 & $\mathrm{p} 27$ & $\begin{array}{c}\text { Cyclin D3 } \\
\text { expression }\end{array}$ & $\begin{array}{c}\mathrm{p} 27 \text { localization } \\
\text { N NC or C }\end{array}$ \\
Normal thyroid & $0 / 4$ & $4 / 4$ & $0 / 4$ & 4 & \\
& $(0 / 1)^{\mathrm{D}}$ & $(1 / 1)$ & $-(0 / 1)$ & $(1)$ & \\
Papillary & $14 / 20$ & $18 / 28$ & $4 / 15$ & 2 & 2 \\
carcinomas & $(2 / 4)$ & $(4 / 4)$ & $-(2 / 4)$ & $(2)$ & \\
& & & $11 / 15$ & 2 & 9 \\
& & & $+(2 / 4)$ & & $(2)$ \\
Follicular & $6 / 7$ & $6 / 9$ & $4 / 4$ & 1 & 3 \\
carcinomas & $(1 / 1)$ & $(1 / 1)$ & $+(1 / 1)$ & $(1)$ & \\
Anaplastic & $3 / 5$ & $2 / 8$ & $2 / 5$ & & $3 / 5^{\mathrm{E}}$ \\
carcinomas & $(2 / 3)$ & $(2 / 3)$ & $-(1 / 3)$ & & $(1 / 3)$ \\
& & & $3 / 5$ & & 2 \\
& & & $+(2 / 3)$ & & $(2)$ \\
\hline
\end{tabular}

Ap27 and cyclin D3 expression was measured by Western blot and quantified by Phosphorlmager. ${ }^{B}$ p 27 expression was considered negative when p 27 protein was less than half the level of normal thyroid. Cyclin D3 expression was considered positive when it was at least 3-fold that of normal thyroid. ${ }^{C} \mathrm{p} 27$ localization was determined by immunostaining and/or by Western blot on differentially extracted proteins. Nuclear localization $(\mathrm{N})$ was assigned when more than $50 \%$ of cells staining posistive for $\mathrm{p} 27$ had nuclear $\mathrm{p} 27$ staining; cytoplasmic localization (C) was assigned when less than $15 \%$ of cells were positive for nuclear p27 and more than $35 \%$ of p27-positive cells had clear cytoplasmic staining; mixed localization (NC) was assigned when $15-50 \%$ of cells that stained for $\mathrm{p} 27$ had simultaneous cytoplasmic and nuclear staining. The same values were used with the intensity of the signal obtained by Western blot. DAll numbers in parentheses refer to cell lines. ${ }^{\mathrm{E}} \mathrm{p} 27$ expression is negative in these tumors and cell lines.

growth of thyroid cells (27). Therefore, we analyzed cyclin D 3 expression by Western blot in 8 cell lines and in 32 thyroid tumors with known p27 expression. We found that cyclin D3 was overexpressed in 23 of 32 tumors and in 4 of 8 tumor cell lines (NIM, NPA, ARO, and FRO), and showed a striking correlation with p27 expression (compare p27 and cyclin D3 expression in Figures $1 \mathrm{a}$ and $2 \mathrm{a}$, respectively).

In addition, we observed that increased cyclin D3 expression correlated with cytoplasmic localization of p27 in thyroid carcinomas (Table 1). In fact, tumors were divided into 2 categories according to cyclin D3 expression (negative or positive) as determined by Western blot, or into 3 subgroups according to p27 localization as determined by immunostaining or Western blot on fractionated extracts (nuclear, cytoplasmic, and mixed), and then plotted. As seen in the plot, tumors or cell lines with cytoplasmic localization of p27 fell into the group with elevated cyclin D3 expression, whereas tumors or cell lines with nuclear p27 localization fell into the group with low cyclin D3 expression, suggesting the existence of a causal relationship between cyclin D3 expression and p27 cytoplasmic localization (Figure 2e).

In normal thyroid HTC-2 cells (with undetectable cyclin D3 expression; Figure 1b), more than $65 \%$ of p 27 was nuclear (not shown). BC-PAP and TPC-1 cells (which expressed low levels of cyclin D3) presented 33\% and $58 \%$ of p 27 as nuclear, respectively. Conversely, NIM, NPA, ARO, and FRO cells (which expressed high 


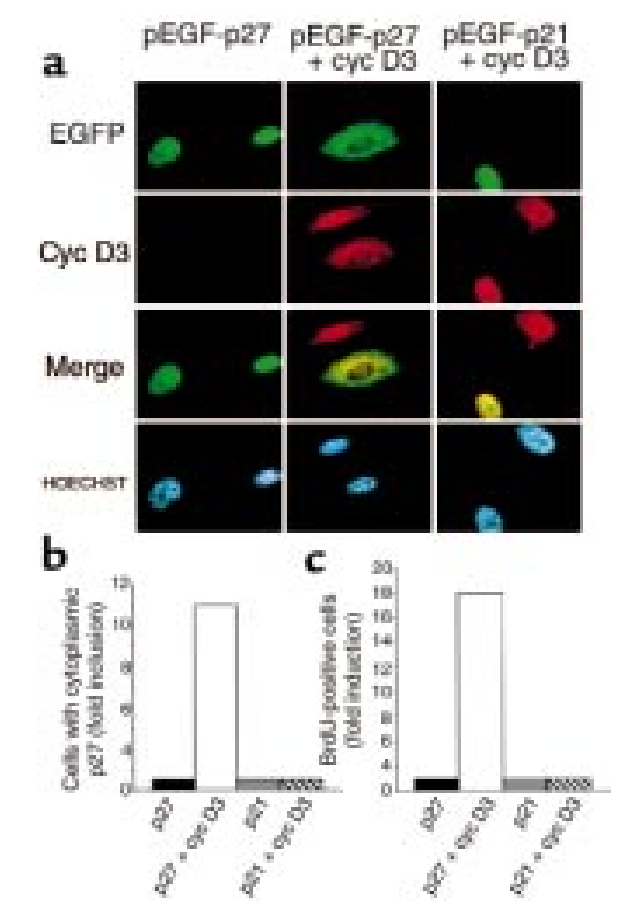

\section{Figure 4}

Cyclin D3 interferes with p27 localization and activity in FB-1 cells. (a) FB-1 cells were transiently transfected as indicated. Localization of p 27 and $\mathrm{p} 21$ was revealed by green fluorescence. Cyclin D3 was revealed by Texas red-conjugated secondary antibodies. (b) Statistical analysis of p 27 and p 21 localization in FB-1 cells. Data represent the mean of 3 different experiments performed in duplicate; at least 200 cells were counted. (c) Statistical analysis of the inhibition of BrdU uptake in FB-1 cells by $\mathrm{p} 27$ and p 21 in the presence or absence of cyclin D3. Data represent the mean of 3 different experiments performed in duplicate; at least 200 cells were counted.

amounts of essentially cytoplasmic cyclin D3) showed the lowest levels of nuclear p27 ( $<20 \%)$, but high levels of cytoplasmic p27. Similar results were obtained from analysis of surgically removed carcinomas. Normal thyroid tissue, which showed undetectable expression of cyclin D3 expression, presented more than $70 \%$ of $\mathrm{p} 27$ as nuclear (Figure 2c, lane NT). Of 4 tumors that showed low expression of cyclin D3 (Figure 2a, lanes 1, 7, 9, and 10), 3 presented low p27 levels (Figure 2a, lanes 1, 9, and 10), whereas the fourth (Figure 2a, lane 7) showed high levels of $\mathrm{p} 27$ protein residing essentially in the nuclear compartment (Figure 2c). Conversely, in all tumors that presented high, essentially cytoplasmic expression of cyclin D3 (Figure 2a, lanes 2 and 4-6), p27 was almost entirely in the cytoplasmic compartment (Figure 2c).

Growth-inhibiting activity exerted by p27 in tumor cells requires nuclear localization. To date, there is no clear information about the relative growth-inhibiting activity of p27 residing in nuclei or cytoplasm. To investigate whether subcellular localization is involved in the regulation of $\mathrm{p} 27$ function, we generated a mutant p27 construct devoid of the nuclear-localization signal (p27NLS) that was unable to enter cell nuclei. In vitro,
p27-NLS bound Cdk2 with the same affinity as did wildtype 27 , and inhibited its activity with apparently the same efficacy (data not shown) (28).

Expression of transfected constructs was determined by Western blotting and immunofluorescence. Localization of the encoded proteins was determined by immunofluorescence or by Western blot on differentially extracted proteins (Figure 3, a-c, e). Both wild-type p27 and p27-NLS proteins were expressed at high levels and localized as expected.

The relative inhibitory activity of p27 and p27-NLS was measured as inhibition percent of BrdU incorporation (IP), calculated as the percentage of transfected cells (green) that incorporated BrdU relative to the percentage of nontransfected cells (not green) that incorporated BrdU. When transfected with pEGFP, FB-1 and Bosc 23 cells continued to incorporate BrdU as did nontransfected cells (IP: $1 \%$ and $2.4 \%$, respectively); BrdU incorporation was reduced by the transfection of wildtype pEGFP-p27 (IP: 94.9\% for FB-1 and 96.7\% for Bosc23 cells, respectively). Conversely, pEGFP-p27NLS exerted a weaker inhibitory effect on the rate of BrdU incorporation (IP: $14 \%$ in FB-1 cells and $39.5 \%$ in Bosc23 cells; Figure 3d).

A representative experiment in FB-1 cells is shown in Figure 3b, with 1 wild-type p27-transfected cell (nuclear localization, red arrow) that did not incorporate BrdU, and a p27-NLS transfected cell (cytoplasmic localization, yellow arrow) that incorporated BrdU. Figure $3 c$ shows a representative experiment in Bosc23 cells. Shown are 3 wild-type p27-transfected cells (nuclear localization, red arrows) that did incorporate BrdU and 1 p27-NLS transfected cell (cytoplasmic, yellow arrow) that incorporated BrdU.

Finally, we investigated whether differences in the level of Cdk2 activity could account for the different growthsuppressing activity exerted by cytoplasm-retained p27NLS and nuclear (wild-type) p27. In Bosc23 cells, wildtype p27 was bound to Cdk2 both in the nucleus and in the cytoplasm, whereas $\mathrm{p} 27-\mathrm{NLS}$ was bound to Cdk2 primarily in the cytoplasmic compartment (Figure 3f). As a result, nuclear Cdk2 activity was reduced in p27transfected cells, whereas it remained elevated in cells transfected with p27-NLS. Because most Cdk2 activity derived from nuclear $\mathrm{Cdk} 2$, the increase in the activity of nuclear Cdk2 due to nuclear exclusion of p27-NLS may account for the increased activity observed in total extracts of p27-NLS-transfected cells. These findings demonstrated that nuclear localization of p27 is necessary for its full inhibitory activity, and provide experimental support for the concept that the high Cdk2 activity observed in tumors with elevated cytoplasmic p27 expression may depend on the inability of p27 to enter cell nuclei and bind Cdk2.

Overexpression of cyclin D3 inhibits $p 27$ translocation from cytoplasm to the nucleus and suppresses $p 27$-induced growth arrest in FB-1 and Bosc 23 cells. To determine the effects exerted by cyclin D3 overexpression on p27 subcellular localization, we used different strategies. First, we used 
a
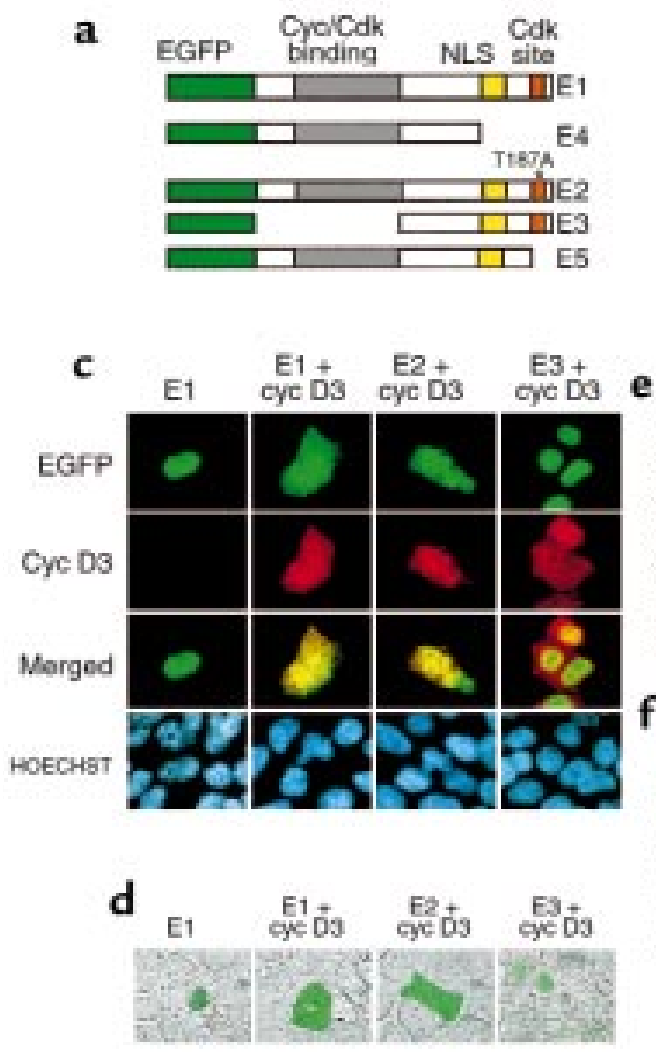
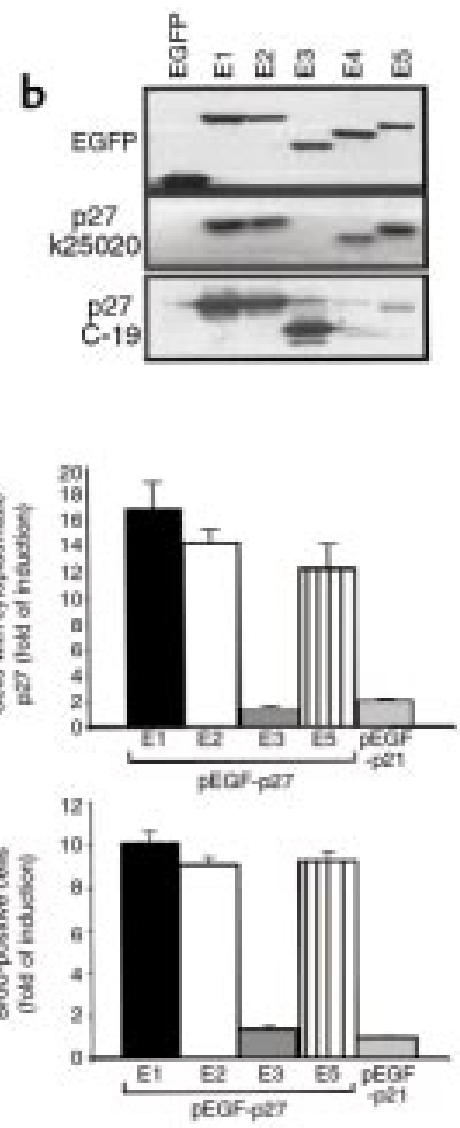

Figure 5

Cyclin D3 interferes with p27 localization and activity in Bosc23 cells. (a) Mutant p27 constructs. (b) Expression of mutant constructs in Bosc23 cells. (c) Immunofluorescence analysis of $\mathrm{p} 27$ localization in Bosc23 cells transfected with the indicated constructs. (d) Immunofluorescence analysis of p27 expression and localization in Bosc23 cells transfected with the indicated construct. Green fluorescence of $p E G F-f u s e d$ proteins was overlaid on the phase-contrast photos to reveal localization. (e) Relative efficacy of cyclin D3 overexpression to sequestrate different p27 mutant constructs in the cytoplasm. (f) Relative efficacy of cyclin D3 overexpression to reverse inhibition of BrdU incorporation induced by the different p27 mutant constructs. E1, p27 (wild-type); E4, p27-NLS; E2, p27TA187; E3, p27-97-197; E5, p27-1-186. a thyroid carcinoma-derived cell line (FB-1) that expresses low levels of endogenous p27 and cyclin D3. FB-1 cells were transiently transfected with pEGF-p27 in the presence of $\mathrm{pCMV}$ or $\mathrm{pCMV}$-cyclin $\mathrm{D} 3$. Immunofluorescence with anti-cyclin D3 antibodies allowed identification of transfected cells. Subcellular localization of $\mathrm{p} 27$ was determined by analysis of green fluorescence emitted by recombinant pEGFP-p27. In the absence of cyclin D3, p27 was found in the nuclear compartment in most cells. When cotransfected with cyclin D3, p27 was found more often (12-fold) in the cytoplasmic compartment (Figure 4b). This effect was dose-dependent. Similar results were obtained when p27 was identified using anti-p27 antibodies. Most important, when pEGF-p21 was coexpressed with cyclin D3, it remained exclusively nuclear. The localization of transfected cyclin D3 was only slightly modified when cotransfected with pEGF-p27, whereas in the case of cotransfection with pEGF-p21, most cyclin D3 became nuclear (compare cyclin D3 localization, Figure $4 \mathrm{a}$ middle and right). Accumulation of p 27 was observed in the cytoplasm after overexpression of cyclin D3 in a different thyroid tumor cell line (TPC-1) that expressed high levels of endogenous nuclear p27 (not shown) and in Bosc 23 cells (Figure 5).

Because they can be transfected with high efficiency, Bosc 23 cells were used as recipient cells to investigate the mechanism of cyclin D3-dependent accumulation of p27 in the cytoplasm. We generated several p27 con- structs (Figure 5a), including p27-TA187, in which a threonine phosphorylated by cyclin E-Cdk2 (which is involved in the regulation of $\mathrm{p} 27$ stability) was changed to alanine (29); p27-97-197, which lacks the cyclinbinding domain; and p27-1-186, which retains the NLS but has lost the threonine 187. All constructs were expressed at comparable levels in Bosc 23 cells, with a transfection efficiency ranging from 35 to $70 \%$, depending on the experiment (Figure 5b). Different antibodies against p27 were used because $\mathrm{k} 25020 \mathrm{did}$ not recognize p27-97-197, and C-19 did not recognize p27-NLS or p27-1-186. Conversely, antibodies to EGFP recognized all recombinant proteins.

When expressed alone, all constructs presented nuclear localization (Figure 5c). With the exception of p27-97-197, all were able to inhibit BrdU incorporation in transfected cells (not shown). When cotransfected in the presence of a 10-fold excess of pCMV-cyclin D3 plasmid ( $0.1 \mu \mathrm{g}$ vs. $1 \mu \mathrm{g})$, we observed that pEGF-p27 (wild-type), pEGF-p27-TA187, and pEGF-p27-1-186 were retained in the cytoplasm of several transfected cells; conversely, pEGF-p27-97-197 remained nuclear in the presence of cyclin D3 (Figure $5, \mathrm{c}-\mathrm{e})$. A construct encoding pEGF-p21 was exclusively nuclear both in the absence and in the presence of cyclin D3 (Figure 5e). Figure 5c shows nuclear localization of pEGF-p27 in the absence of cyclin D3, and nucleocytoplasmic localization of pEGF-p27 and pEGF-p27-TA187 in the presence of cyclin D3. In the 

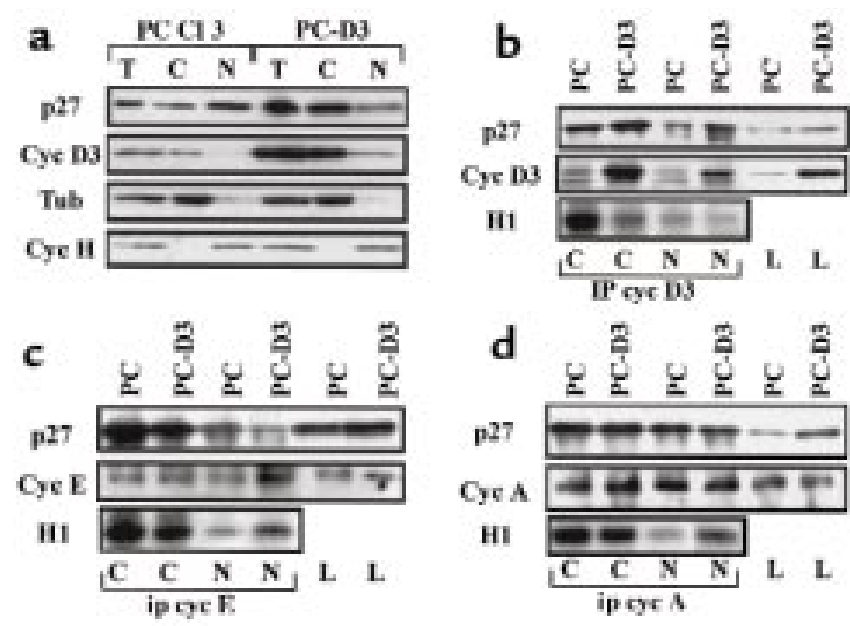

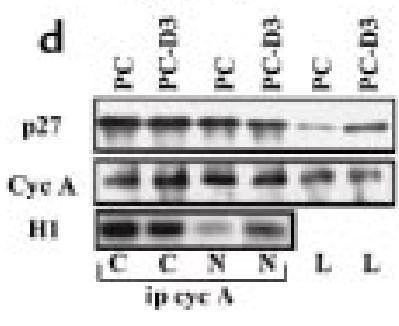

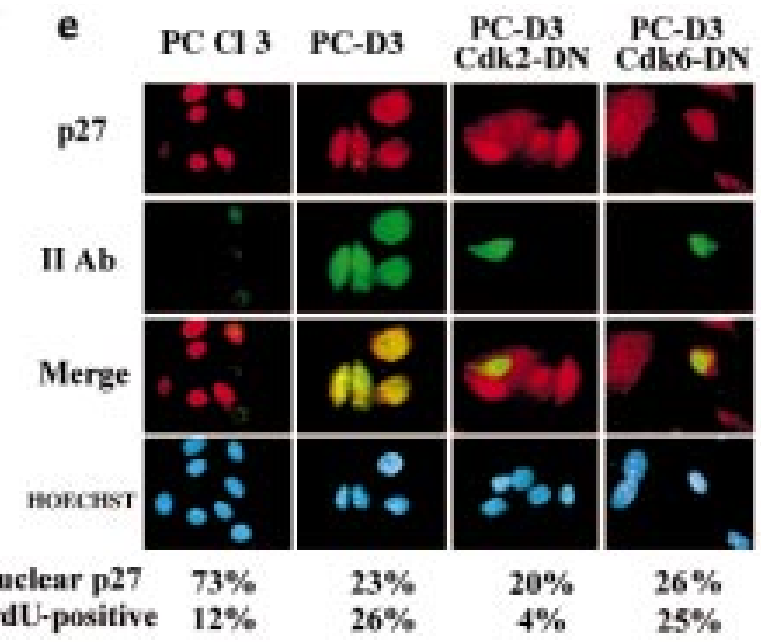

\section{Figure 6}

Cyclin D3 induces p27 retention in the cytoplasmic compartment in PC-D3 cells. (a) Western blot analysis of p27 and cyclin D3 in PC Cl 3 and PC-D3 cells. Total cell lysates (T), cytoplasmic fractions (C), and nuclear fractions (N) were analyzed. (b-d) Analysis of cytoplasmic and nuclear complexes. Immunoprecipitates of (b) cyclin D3, (c) cyclin E, and (d) cyclin A. (e) Immunofluorescence analysis of p27 in PC Cl 3 and PC-D3 cells. Cells were stained with anti-p27 antibodies and revealed with Texas red-conjugated antibodies (top). Cells were stained with anti-cyclin D3 antibodies (first and second columns) or anti-HA (third and fourth columns) and revealed with FITC-conjugated antibodies (II Ab; second row). Overlapping of red and green staining is shown in the third row. HOECHST staining of cell nuclei appears in the fourth row. Each experiment was repeated 3 times; at least 200 cells were counted. Statistical results for p 27 nuclear localization and BrdU incorporation are reported at the bottom. $\times 100$

presence of cyclin D3, pEGF-p27-97-197 (which lacks the cyclin-binding domain) also remained nuclear. In Figure $5 \mathrm{~d}$, the green fluorescence emitted by EGF-p27 proteins was mounted onto contrast-phase photos to reveal p27 localization.

Similar results were obtained with transfection of a plasmid encoding cyclin D1 (not shown), suggesting that all $\mathrm{D}$ cyclins may be involved in the regulation of p27 localization. However, cyclin D1 shifted p27 with reduced efficacy compared with cyclin D3.

Finally, we investigated whether cyclin D3 overcomes p27-induced growth arrest in cells in which cyclin D3 induced cytoplasmic retention of $\mathrm{p} 27$. When expressed in FB-1 cells in the presence of pCMV alone, p27 inhibited $\mathrm{BrdU}$ incorporation by almost $95 \%$. In the presence of cyclin D3, the inhibitory effect of p27 was reduced (Figure 4c). In Bosc23 cells, cyclin D3 - which alone had no effect on the rate of BrdU incorporation increased BrdU incorporation more than 16-fold when cotransfected with pEGF-p27 (wild-type), p27-TA187, or p27-1-186 (Figure 5f). As with localization, expression of cyclin D3 was not able to reverse the block in BrdU incorporation induced by pEGF-p21. The ability of cyclin D3 to abrogate p27-mediated cell-cycle inhibition was greater than its ability to alter localization of p27. Therefore, changing p27 localization may be only one of the mechanisms used by cyclin D3 to enhance tumor-cell growth.

Effects of cyclin D3 overexpression on the localization, composition, and activity of p27-containing complexes in thyroid cells. Finally, we made use of 3 stable clones of a normalthyroid rat cell line, the $\mathrm{PC} \mathrm{Cl} 3$ cells, which were engineered to overexpress cyclin D3 (clones 1, 5, and 6). PC
$\mathrm{Cl} 3$ cells expressed high amounts of $\mathrm{p} 27$, localized predominantly in the nucleus. PC-D3 cells showed higher levels of p27 than did parental cells, but p27 protein was increased 2.3-fold in the cytoplasm and reduced by half in the nucleus (Figure 6a). Results obtained by Western blot were confirmed by immunofluorescence. PC Cl 3 cells showed low cyclin D3 and strong nuclear p27 immunoreactivity (> 70\% of cells) (Figure 6e, left row). Conversely, PC-D3 cells showed high cyclin D3 immunoreactivity in both the cytoplasmic and the nuclear compartments and a much-reduced number of cells with nuclear p27 staining (23\%). Most cells presented nucleocytoplasmic p27 localization, with fewer cells showing exclusive cytoplasmic localization (Figure $6 e$, second column). PC-D3 cells presented increased BrdU incorporation compared with parental cells $(26 \%$ vs. $12 \%$, respectively).

To analyze the composition of p27-containing nuclear and cytoplasmic complexes in PC-D3 cells, fractionated proteins from $\mathrm{PC} \mathrm{Cl} 3$ and PC-D3 cells were immunoprecipitated with antibodies against Cdk2, Cdk6, cyclin D3, cyclin E, and cyclin A. Cells were subsequently analyzed by Western blot using antibodies against p27, cyclin D3, cyclin E, and cyclin A for Cdk immunoprecipitates or antibodies against p27, Cdk2, Cdk4, and Cdk6 for cyclin immunoprecipitates. All immunoprecipitates were normalized by determining the levels of the immunoprecipitated proteins by Western blot. Analysis of Cdk immunoprecipitates demonstrated that in PC-D3 cells, the amount of cyclin D3 bound to cytoplasmic Cdk2 and Cdk6 was increased (7- and 3.8-fold, respectively, not shown). In addition, in PC-D3 cells there was a marked increase in $\mathrm{p} 27$ bound to cytoplas- 
mic cyclin D3 (Figure 6b) and Cdk2, and only a slight increase in the amount bound to Cdk6 (not shown). Conversely, we found decreased amounts of p 27 bound to nuclear cyclin $\mathrm{E}$ and cyclin A (3- and 1.8-fold, respectively) (Figure 6, c and d), which probably resulted from the recruitment of p27 into cytoplasmic cyclin D3-Cdk complexes. Kinase assays using GST-RB or histone H1 as substrates demonstrated that Cdk2 activity was increased in the nuclear fraction (almost 3-fold) of PCD3 compared with PC Cl 3 cells (not shown). The increase in nuclear Cdk2 activity was accompanied by a parallel increase in activity of kinases associated with cyclin A and with cyclin E (2.2- and 3-fold, respectively) (Figure 6, c and d).

Because in thyroid cells cyclin D3 associated essentially with Cdk2 and Cdk6, we tested whether activation of Cdk2 and Cdk6 was necessary for cyclin D3-mediated p27 cytoplasmic retention. We transiently transfected PC-D3 cells with plasmids encoding dominant-negative mutants of Cdk2 or Cdk6 (Cdk2$\mathrm{DN}$ and Cdk6-DN, respectively) and determined p27 localization by immunofluorescence. We used Cdk2DN with a D145N mutation and Cdk6-DN with a D163N mutation (30). As shown in Figure 6e, the localization of p27 in PC-D3 cells in which Cdk2 or Cdk6 activity had been impaired by use of dominant-negative constructs remained essentially nucleocytoplasmic or cytoplasmic, respectively, as it did in nontransfected PC-D 3 cells, suggesting that activity of kinases in the ternary cyclin D3- and Cdk-p27 complexes (i.e., phosphorylation of p27) was not required.

\section{Discussion}

In this study, we provide evidence of the existence of at least 2 different mechanisms whereby the activity of the Cdk inhibitor p27 is overcome in thyroid cancer cells. The expression of $\mathrm{p} 27$ protein is reduced in most poorly differentiated thyroid carcinomas, but only in a subset of well-differentiated carcinomas, of both the papillary and follicular histotypes. Surprisingly, several tumors that retained p27 expression presented high Cdk2 activity, raising the question of how tumor cells can escape p27 inhibition. Our results indicate that a major difference between normal and malignant thyroid cells was in the subcellular localization of p27. In normal thyrocytes, more than $70 \%$ of $\mathrm{p} 27$ was detected in the nuclear compartment, whereas in tumor cells, p27 was essentially confined to the cytoplasmic compartment. Cytoplasmic localization of p27 has been reported previously $(25,26)$, but in those studies no functional implication was provided. In this paper we address the biological significance of p27 residing in the nucleus or the cytoplasm. Using a mutant p27 devoid of its $\mathrm{COOH}$-terminal nuclear-localization signal (p27-NLS), we were able to prevent p27 nuclear translocation and thus to reproduce, at least in part, the situation found in several thyroid tumors and tumor cell lines (i.e., high cytoplasmic expression of p27 but very low nuclear levels). When compared with wild-type p27, p27-NLS was much less efficient in inhibiting progression to the $S$ phase of the cell cycle in 2 different cell lines, which probably resulted from the inability of cytoplasm-retained p27 to bind and inhibit nuclear Cdk2, as is the case with wild-type p27.

On the basis of this observation we would expect that in thyroid tumors, Cdk2 activity would be inversely correlated with the amount of nuclear Cdk2-bound p27. Indeed, in a representative panel of thyroid tumorderived cell lines and surgically removed carcinomas, samples with low Cdk2 activity presented higher levels of 227 bound to nuclear $\mathrm{Cdk} 2$ than did samples with high Cdk2 activity, suggesting that availability of $\mathrm{p} 27$ (i.e., subcellular localization) is more important than its absolute amount in regulating its function.

Moreover, our results indicate that in most thyroid tumors and tumor-derived cell lines, cyclin D3 presents nucleocytoplasmic or cytoplasmic localization that corresponds to p27 localization. Therefore we investigated whether the cytoplasmic retention of p27 that was observed in thyroid tumors could be caused by formation of cytoplasmic complexes induced by expression of cyclin D3. We tested this model by transient cotransfection experiments and by generation of a cyclin D3-overexpressing thyroid cell line (PC-D3). Cytoplasmic accumulation of $\mathrm{p} 27$ caused by cyclin D3 overexpression was observed in both cases. Consistent with the concept that nuclear localization is necessary for inhibition of p27 activity, cyclin D3 rescued p27-imposed G1 phase arrest and induced increased the growth rate in stably transfected cells. Because cyclin D1 also shifted p27 localization (albeit with reduced efficacy compared with cyclin D3), it is likely that all D cyclins are involved in regulating $\mathrm{p} 27$ localization. This was not unexpected, because cyclin D1 was shown to override a p27-dependent G1 block in NIH 3 T3 cells without reducing the level of p27 protein as does cyclin E (30). Accordingly, in PC-D3 cells, p27 expression was not reduced, but it accumulated predominately in the cytoplasm.

In thyroid-transformed cells, cyclin D-Cdk complexes acted as reservoirs of $\mathrm{p} 27$, regulating its availability to complexes containing cyclin $\mathrm{E}$ or cyclin $\mathrm{A}$, as has been previously reported (10). However, in this case such complexes were physically separated, as observed in anchorage-independent rat fibroblasts (31). Accordingly, in PC-D3 cells, p27 recruitment into cytoplasmic cyclin D3-Cdk2 complexes resulted in the titration of p27 from nuclear cyclin A-Cdk2 and cyclin E-Cdk2 complexes. As a consequence of complex shuffling, the level of $\mathrm{p} 27$ bound to nuclear Cdk2 decreased in parallel with an increase in activity of Cdk2 bound to nuclear cyclin A and cyclin E, reproducing the situation observed with the expression of p27-NLS.

D cyclins lack nuclear-localization signals that allow their entry into the nucleus, but their nuclear translocation may occur after they bind to Cdk inhibitors or Cdk's $(29,32)$. However, nuclear targeting of cyclin D3-Cdk complexes by $\mathrm{p} 27$ does not exclude the possibility that highly expressed cyclin D3 may form cyto- 
plasmic complexes that contribute to retention of $\mathrm{p} 27$ in the cytoplasm. In support of this concept, p27 is much less efficient in targeting cyclin $\mathrm{D}-\mathrm{Cdk}$ complexes to the nucleus than is p21 $(29,32,33)$. On the other hand, as we have shown here, cyclin D3-dependent shift in subcellular localization occurred more efficiently with p27 than p21. Cytoplasmic retention of cyclin D3- and Cdk-p27 complexes could be due to the presence of a specific cytoplasmic signal in cyclin D3 that is dominant over the nuclear-localization signal, as is the case with cyclin B-Cdk1 (34). Alternatively, this retention could be caused by sequestration of p27 in a form that is unable to bind cyclin E-Cdk2 (as occurs in c-Myc overexpression; ref. 35), or by association with a specific cytoplasmic-retention protein, as is the case of Jun $\mathrm{N}$-terminal kinase (36).

Experiments with p27 mutants and with dominantnegative Cdk2-DN and Cdk6-DN indicated that cyclin D3-dependent shifting of p27 required that cyclin D3 bind to $\mathrm{p} 27$, although kinase activity of the complex was not required. In fact, the p27 mutant lacking the cyclinbinding domain was not shifted, whereas p27-TA187 (the p27 mutant in which the threonine phosphorylated by Cdk 2 was replaced by alanine) was shifted with the same efficacy as was wild-type p27. Finally, inhibition of Cdk2 or Cdk6 activity did not interfere with cyclin D3-dependent p27 cytoplasmic accumulation.

In conclusion, our results allowed us to propose a model whereby inactivation of p27 in thyroid tumors may occur either by p27 downregulation or by cytoplasmic sequestration, because cytoplasm-residing p27 is inactive as a growth inhibitor. In thyroid tumors, cyclin D3 overexpression may account, at least in part, for p27 cytoplasmic localization. However, further studies are necessary to elucidate the mechanisms whereby p27 is recruited into cytoplasmic complexes and whether this takes place only in thyroid carcinoma cells or also occurs in other tumors.

\section{Acknowledgments}

This work was supported by grants from the Associazione Italiana Ricerca sul Cancro and Progetto Finalizzato Biotecnologie of the Consiglio Nazionale delle Ricerche, to G. Viglietto and A. Fusco. G. Baldassarre, B. Belletti, P. Bruni, F. Trapasso, and A. Boccia are supported by Federazione Italiana per la Ricerca sul Cancro fellowships. We are indebted to M. Santoro for providing Bosc 23 cells, and to G. Draetta for the cyclin D1 and the Cdk2-DN plasmids.

1. Hedinger, C., Williams, E.D., and Sobin, L.H. 1989. The WHO histological classification of thyroid tumors: a commentary on the second edition. Cancer. 63:908-911.

2. Fearon, E.R., and Vogelstein, B. 1990. A genetic model for colorectal tumorigenesis. Cell. 61:759-767.

3. Wynford-Thomas, D. 1993. Molecular basis of epithelial tumorigenesis: the thyroid model. Crit. Rev. Oncog. 4:1-23.

4. Grieco, M., et al. 1990. PTC is a novel rearranged form of the ret protooncogene and is frequently detected in vivo in human thyroid papillary carcinomas. Cell. 60:557-563.
5. Namba, H., Rubin, S.A., and Fagin, J.A. 1990. Point mutations of ras oncogenes are an early event in thyroid tumorigenesis. Mol. Endocrinol. 4:1474-1479

6. Fagin, J.A., et al. 1993. High prevalence of mutations of the p53 gene in poorly differentiated human thyroid carcinomas. J. Clin. Invest. 91:179-184. 7. Sherr, C.J. 1994. G1 phase progression: cycling on cue. Cell. 79:551-555. 8. Weinberg, R.A. 1995. The retinoblastoma protein and cell cycle control. Cell. 81:323-330.

9. Sherr, C.J. 1993. Mammalian G1 cyclins. Cell. 73:1059-1065.

10. Sherr, C.J., and Roberts, J.M. 1995. Inhibitors of mammalian G1 cyclindependent kinases. Genes Dev. 9:1149-1163.

11. el-Deiry, W.S., et al. 1993. WAF1, a potential mediator of p53 tumor suppression. Cell. 75:817-825.

12. Polyak, K., Lee, M., Koff, A., Roberts, J.M., and Massaguè, J. 1994. Cloning of p27kip-1, a cyclin-dependent kinase inhibitor and potential mediator of extracellular antimitogenic signals. Cell. 78:59-66.

13. Lee, M.H., Reynisdottir, I., and Massaguè, J. 1995. Cloning of $\mathrm{p} 57^{\mathrm{KIP} 2}$, a cyclin-dependent kinase inhibitor with unique domain structure and tissue distribution. Genes Dev. 9:639-649.

14. Nakayama, K., et al. 1996. Mice lacking p27(kip1) display increased body size, multiple organ hyperplasia, retinal dysplasia, and pituitary tumors. Cell. 85:707-721.

15. Catzavelos, C., et al. 1997. Decreased levels of the cell-cycle inhibitor p27kip1 protein: prognostic implications in primary breast cancer. Nat. Med. 3:227-230.

16. Loda, M., et al. 1997. Increased proteasome-dependent degradation of the cyclin-dependent kinase inhibitor p27 in aggressive colorectal carcinomas. Nat. Med. 3:231-234.

17. Esposito, V., et al. 1997. Prognostic role of the cyclin-dependent kinase inhibitor p27 in non-small cell lung cancer. Cancer Res. 57:3381-3385.

18. Erickson, L.A., et al. 1998. Expression of p27kip1 and Ki-67 in benign and malignant thyroid tumors. Mod. Pathol. 11:169-174.

19. Resnick, M.B., Schacter, P., Finkelstein, Y., Kellner, Y., and Cohen, O. 1998. Immunohistochemical analysis of p27/kip 1 expression in thyroid carcinoma. Mod. Pathol. 11:735-739.

20. Yaroslavskiy, B., Watkins, S., Donnemberg, A.D., Patton T.J., and Steinman, R.A. 1999. Subcellular and cell-cycle expression profiles of Cdk inhibitors in normal differentiating myeloid cells. Blood. 93:2907-2917.

21. Battista, S., et al. 1998. Increase in AP-1 activity is a general event in thyroid cell transformation in vitro and in vivo. Oncogene. 17:377-385.

22. Pagano, M., et al. 1993. Regulation of the cell cycle by the cdk2 protein kinase in cultured human fibroblasts. J. Cell Biol. 121:101-111.

23. Baldassarre, G, et al. 1999. Key role of the cyclin-dependent kinase inhibitor p27kip1 for embryonal carcinoma cell survival and differentiation. Oncogene. In press.

24. Motokura,T., Keyomarsi, K., Kronenberg, H.M., and Arnold, A. 1992. Cloning and characterization of human cyclin D3, a cDNA closely related in sequence to the PRAD1/cyclin D1 proto-oncogene. J. Biol. Chem. 267:20412-20415.

25. Ciaparrone, M., et al. 1998. Localization and expression of p27KIP1 in multistage colorectal carcinogenesis. Cancer Res. 58:114-122.

26. Singh, S.P.J., et al. 1998. Loss or altered subcellular localization of p27 in Barrett's associated adenocarcinoma. Cancer Res. 58:1730-1735.

27. Depoortere, F., et al. 1998. A requirement for cyclin D3-cylin-dependent kinase (cdk)-4 assembly in the cyclic adenosine monophosphatedependent proliferation of thyrocytes. J. Cell Biol. 140:1427-1439.

28. Font de Mora, J., Uren, A., Heidaran, M., and Santos, E. 1997. Biological activity of p27 and its amino- and carboxy-terminal domains in G2/M transition of Xenopus oocytes. Oncogene. 15:2541-2551.

29. Sheaff, R., Groudine, M., Gordon, M., Roberts, J.M., and Clurman, B.E. Cyclin E-Cdk2 is a regulator of p27. 1997. Genes Dev. 11:1464-1478.

30. Van den Heuvel, S., and Harlow, E. Distinct role of cyclin-dependent kinases in cell cycle control. 1993. Science. 262:2050-2053.

31. Orend, G., Hunter, T., and Ruoslahti, E. 1998. Cytoplasmic displacement of cyclin E-cdk2 inhibitors p21Cip1 and p27Kip1 in anchorage-independent cells. Oncogene. 16:2575-2583.

32. LaBaer, J., et al. 1997. New functional activities for the p21 family of Cdk inhibitors. Genes Dev. 11:847-862.

33. Reynisdottir, I., and Massagué, J. 1997. The subcellular locations of p15 and p27 coordinate their inhibitory interactions with Cdk4 and Cdk2. Genes Dev. 11:492-503.

34. Pines, J., and Hunter, T. 1994. The differential localization of human cyclins A and B is due to a cytoplasmic retention signal in cyclin B. EMBO J. 13:3772-3781.

35. Vlach, J., Hennecke, S., Aleviozopoulos, K., and Amati, B. 1996. Growth arrest by the cyclin-dependent kinase inhibitor $\mathrm{p} 27$ is abrogated by $\mathrm{c}$ Myc. EMBO J. 15:6595-6604.

36. Dickens, M., et al. 1997. A cytoplasmic inhibitor of the JNK signal transduction pathway. Science. 277:693-696. 